\title{
Influence of Positron Irradiation on Positronium Formation in $\boldsymbol{n}$-Alkanes
}

\author{
M. Pietrow* AND B. ZgardzińsKA
}

Department of Nuclear Methods, Institute of Physics

M. Curie Skłodowska University

pl. Marii Curie Skłodowskiej 1, 20-031 Lublin, Poland

\begin{abstract}
Positronium formation probability was investigated as a function of irradiation time and temperature for some long-chain saturated hydrocarbons (alkanes). Intensity of ortho-positronium component in the lifetime spectrum depends on the concentration of trapped excess electrons, but also on concentration of radicals and ions acting as electron scavengers. The results indicate the existence of two kinds of electron traps; those which are not emptied by thermal treatment disappear after several days of positron irradiation.
\end{abstract}

PACS numbers: 36.10.Dr, 61.80.Fe, 78.70.Bj

\section{Introduction}

The positronium (Ps) formation in the matter consists essentially in the capture of the quasi-free electron by the positron. Details of this process are described by the spur model $[1,2]$ assuming that positron combines with one of electrons from the last ionization product cluster ("blob"). However, it has been noticed recently $[3,4]$ that with a prolonged duration of experiment, at low temperatures, the probability of the Ps formation (observed via triplet ortho-Ps intensity) rises. The electrons which have been knocked by positron along its ionization track diffuse and locate in intermolecular cavities which are electron traps about $0.5-2 \mathrm{eV}$ deep. These traps become populated during the time of experiment and serve as an extra reservoir of electrons, capable to form a positronium if the Ps binding energy is greater than the trap depth. The electrons located in such traps may be removed by illumination of the sample by the light with a sufficient quantum energy. The influence of light on Ps formation in polymers at low temperatures has been studied by Hirade et al. [5-7]. Other processes which influence the Ps formation are pointed out in Refs. $[8,9]$. In those papers the role of radicals,

*corresponding author; e-mail: mrk@kft.umcs.lublin.pl 
which compete with positrons in picking the excess electrons, is stressed. Also the functional dependence of the Ps intensity against the time is elaborated there in detail. Authors have shown that the familiar three-exponential decomposition (para-Ps and ortho-Ps formation, and the free annihilation of positrons) of the experimental lifetime spectrum is only an approximation of more complicated behavior of positronium intensity in time. Each of such three components has the decay constant nontrivially dependent on time because of the existence of much subtle processes which have been usually neglected. These processes are, for example, capture of electrons and positrons by ions and radicals, migration of the excess electrons to the traps, outdiffusion of positrons from the blob region, etc.

Up to now the bulk of the papers on the influence of irradiation on the positronium formation concerns polymers. In this paper we describe the investigations of the $o$-Ps formation in some polycrystalline alkane and alcohol samples which have, comparing to polymers, a less complex structure.

\section{Experimental}

The samples of $n$-undecane $\mathrm{C}_{11} \mathrm{H}_{24}, n$-tridecane $\mathrm{C}_{13} \mathrm{H}_{28}$, and $n$-heptadecane $\mathrm{C}_{17} \mathrm{H}_{36}$ were placed in the container with a positron source $\left({ }^{22} \mathrm{Na}\right.$, about $\left.0.15 \mathrm{MBq}\right)$ in a Kapton envelope fixed in the center. Liquid samples were degassed by the freeze-thaw method. The container was fixed to the upper end of cold finger contacting with liquid nitrogen and a heating coil was placed just below the container. The temperature was regulated with an accuracy of $\pm 0.1 \mathrm{~K}$ by Shimaden FP21 regulator with $\mathrm{T}$ type thermocouple. The chamber was evacuated to the air pressure of about $0.5 \mathrm{~Pa}$ to avoid ortho-para conversion in the presence of molecular oxygen. Two light emitting diodes inside the container could illuminate the sample. The wavelength of light was $\approx 0.95 \mu \mathrm{m}$, which is sufficiently short to make empty the shallow traps accommodating excess electrons. The positron lifetime spectra were registered using a standard fast-slow delayed coincidence spectrometer. Two counters with $\mathrm{BaF}_{2}$ crystals were placed at an angle of $90^{\circ}$ between their axes. Each positron lifetime spectrum was collected during $1 \mathrm{~h}\left(3.6 \times 10^{5}\right.$ counts per spectrum). Deviation from exponentiality, mentioned in the Introduction, appears in the first $0.3 \mathrm{~ns}$ after positron birth [9], while we were interested mainly in lifetime and intensity of relatively long-lived ortho-positronium, thus we neglected that deviation and analyzed the spectra by LT programme [10] as a sum of 3 discrete exponential components convoluted with a resolution curve (resolution time was about $240 \mathrm{ps}$ ). The components were ascribed to the decay of singlet para-Ps, annihilation of free positrons, and the decay of ortho-Ps; the correction for positron absorption in Kapton envelope was also applied.

\section{Results and discussion}

The effect of ortho-Ps intensity $I_{3}$ rise in time was investigated in the case of $n$-undecane and $n$-tridecane, additional measurements were done for 


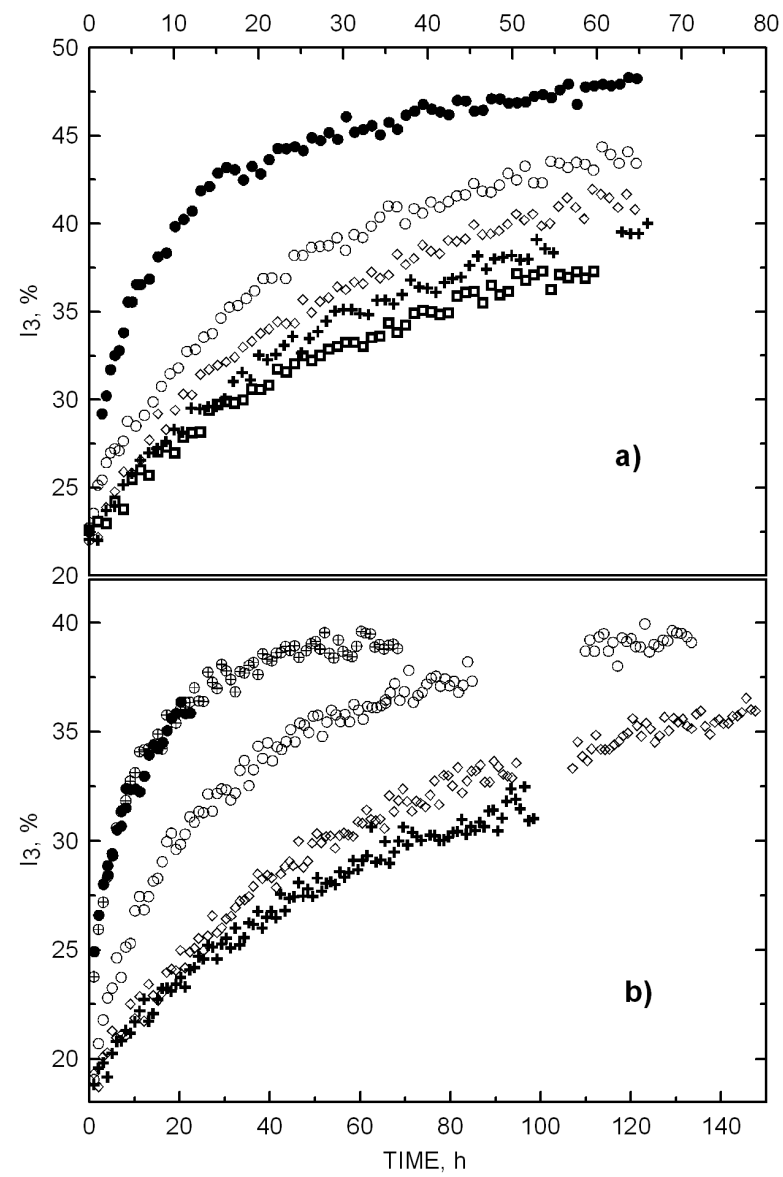

Fig. 1. Ortho-Ps intensity at $125 \mathrm{~K}$ as a function of time; irradiation by positron source. 1st cycle — dots, 2nd - open circles, 3rd — diamonds, 4th — crosses, 5th — squares, circles with cross - the cycle after sample melting and 2 months storage at RT without positron source. (a) $n$-undecane, (b) $n$-tridecane.

$n$-heptadecane. Degassed liquid samples of undecane (melting point $249 \mathrm{~K}$ ) were cooled to the temperature of $125 \mathrm{~K}$ and in one hour intervals the lifetime spectra were recorded. After 64 hours the infrared emitting diodes were switched on for $8 \mathrm{~h}$ and trapped electrons were removed. Then, the cycle of measurement in the darkness, followed by illumination, was repeated four times. The results for undecane are shown in Fig. 1a. We have not observed the decrease of intensity in time which is seen sometimes in polymers [7]. Every next cycle the saturation value of $I_{3}$ is lower. In the first run the $I_{3}(t)$ curve can be approximated by a sum of two exponential functions

$$
N(t)=I_{3 \max }-A \exp \left(-t / \theta_{1}\right)-B \exp \left(-t / \theta_{2}\right),
$$


where $I_{3 \max }$ is the saturation value of total intensity, $A$ and $B$ are the partial intensities of "trap components", $\theta_{1}$ and $\theta_{2}$ are the time constants of $o$-Ps rise ( $I_{3 \max }-A-B$ is the intensity without irradiation). In next cycles one exponential with a longer time constant is enough. It means that the mechanism of rapid picking disappears in a time shorter than 65 hours. Table shows the fitted values of saturation intensity $I_{3 \max }$ and time constants $\theta_{i}$ of $I_{3}$ rise. It is seen that the time constant weakly depends on irradiation time (except the first run). The lack of one of exponential processes after the first irradiation cycle can be related to the destruction of one type of electron traps. This explanation however requires a crucial experiment in the future.

\section{TABLE}

Fitted values of saturation intensity $I_{3 \max }$ and time constants $\theta_{i}$ of $I_{3}$ growth for the sequence of measuring cycles for $n$-undecane and $n$-tridecane at $125 \mathrm{~K}$.

\begin{tabular}{c|c|c|c}
\hline \hline Cycle no. & $I_{3 \max }, \%$ & $\theta_{1}, \mathrm{~h}$ & $\theta_{2}, \mathrm{~h}$ \\
\hline \multicolumn{4}{c}{$n$-undecane, $\mathrm{C}_{11} \mathrm{H}_{24}$} \\
\hline 1 & 53.4 & 6.0 & 80.6 \\
2 & 44.6 & 21.0 & - \\
3 & 43.1 & 26.4 & - \\
4 & 41.5 & 29.0 & - \\
5 & 40.3 & 32.7 & - \\
\hline \multicolumn{5}{c}{$n$-tridecane, $\mathrm{C}_{13} \mathrm{H}_{28}$} \\
\hline 1 & 39.4 & 3.6 & 17.2 \\
2 & 38.9 & 28.6 & - \\
3 & 37.0 & 56.5 & - \\
4 & 34.1 & 55.2 & -
\end{tabular}

Diminishing the rate of $I_{3}$ increase and decrease in asymptotic $I_{3}$ value can be explained in two ways:

- the number of traps for electrons decreases with time elapse,

- electrons produced in the ionization track are scavenged by other ionization products (radicals, ions, etc.). The density of radicals rises in time and thus, the number of electrons reaching the traps decreases.

The second variant seems much more probable.

Similar measurements (with the same positron source) were done for $n$-tridecane (Fig. 1b) and the results were identical as for $n$-undecane (reduction of saturation intensity, shape of $I_{3}$ dependence on time). Time constants $\theta_{i}$ in that case were larger than in undecane, thus one cycle of measurement lasted $150 \mathrm{~h}$. 
After about two months of measurements (and continuous irradiation by positrons) the sample of $n$-tridecane was stored in liquid phase for 2 months, placed again in the container with positron source and one run of the measurements was repeated. It is seen in Fig. 1b that the $I_{3}$ dependence on time was restored, the sample behaved like nonirradiated one. It can be the result of real regeneration of sample structure, or homogenization of sample. The range of positrons in the sample is below $1 \mathrm{~mm}$, thus a small part of the sample is irradiated and all defects are confined in a small volume; after melting the sample is well mixed and the region near the source is again poor in irradiation products. No effect of $I_{3}$ rise is observed in the rotator and liquid phases.

The temperature dependence of $I_{3}$ was also investigated. Fresh (nonirradiated) sample of $n$-undecane was kept at $125 \mathrm{~K}$ up to the moment when the intensity approached the saturation value and then the temperature was increased in steps. These values of $I_{3}$ have been compared with those for the sample which, at the temperature of $125 \mathrm{~K}$, was irradiated during $40 \mathrm{~h}$, illuminated and kept further $26 \mathrm{~h}$ to reach again the saturation of intensity. One observes (Fig. 2, open circles) not only the reduction of $o$-Ps intensity, but also the shift of $I_{3}(T)$ curve toward lower temperatures. At the temperature of about $200 \mathrm{~K}$ the intensity $I_{3}$ begins to decrease, which is commonly explained as the result of escape of trapped electrons due to the activation of thermal motion of molecular end groups. That

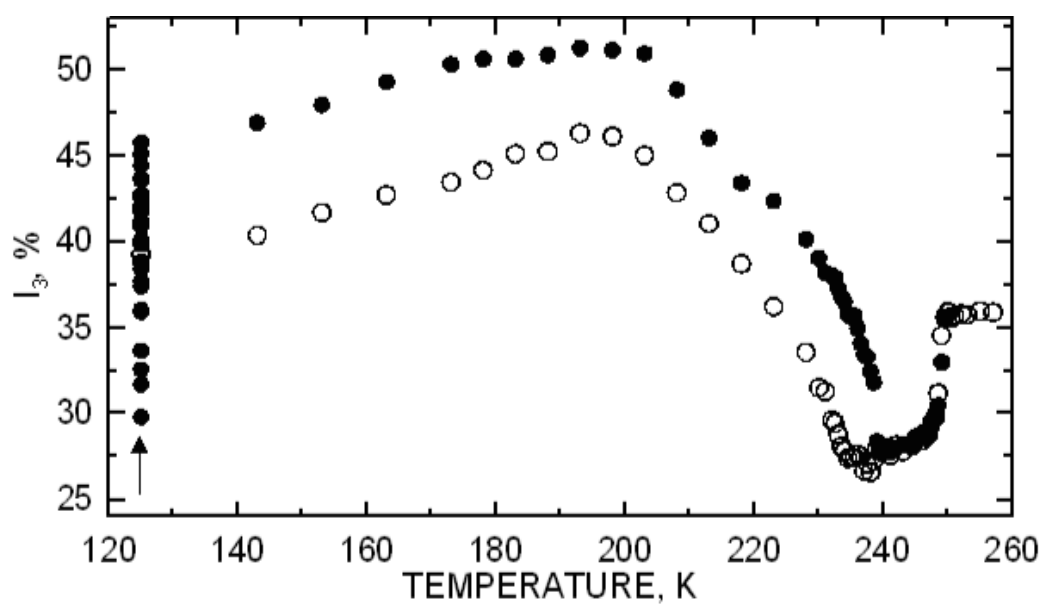

Fig. 2. $o$-Ps intensity in $n$-undecane as a function of temperature. Dots - without preliminary irradiation; open circles — after $66 \mathrm{~h}$ storage (irradiation) at $125 \mathrm{~K}$.

startpoint of $I_{3}$ decrease is very similar for all alkanes studied yet. In $n$-undecane the region of $I_{3}(T)$ decrease extends up to the transition to the rotator phase, but in the case of longer chains there is a range of temperatures where, after initial drop, the intensity remains almost constant, nevertheless it is higher than that observed in illuminated samples. It was noticed earlier for very long chains [11] 
that directly after cooling from the rotator phase the $o$-Ps intensity in that range of temperatures rises, with time constant of several hours. That increase amounts several percent only. One can suppose that there exists another kind of traps (we denote them below as "traps B"), whose activity is eliminated by light, but not by an increase in temperature (in Ref. [11] they were observed even at $320 \mathrm{~K}$ ). It seemed interesting to see how these traps behave during long-time irradiation. In $n$-heptadecane the region of dominant traps B is located between $250 \mathrm{~K}$ and $280 \mathrm{~K}$. The $I_{3}$ vs. time dependence was measured at $273 \mathrm{~K}$. The $I_{3}$ level with the light on is about $22 \%$, the $I_{3}$ values as a function of time (time zero is the moment of cooling from liquid to $273 \mathrm{~K}$ ) are shown in Fig. 3. The initial rise is followed by a decrease, down to the "light on" level, or even lower, to $21 \%$, after $230 \mathrm{~h}$ of measurement.

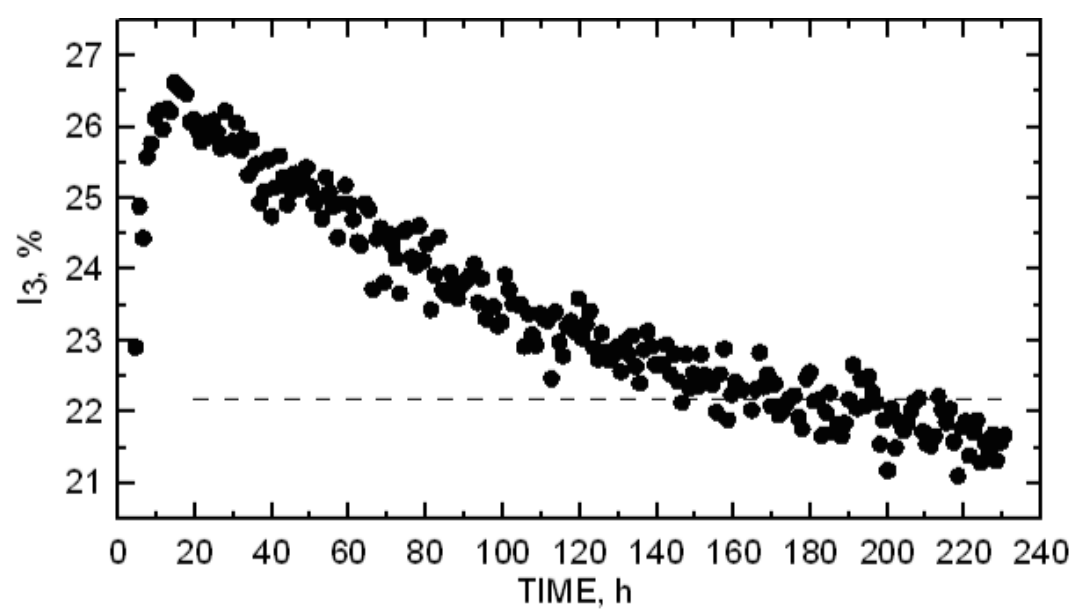

Fig. 3. o-Ps intensity in $n$-heptadecane as a function of time directly after cooling from liquid phase to $273 \mathrm{~K}$. Dashed line shows the $I_{3}$ intensity in fresh, illuminated sample.

That total elimination of electrons in the traps B was not expected. In tridecane at $130 \mathrm{~K}$ the reduction of $o$-Ps formed with excess electrons is only partial, even after 600 hours of irradiation. So, there are two possibilities:

- effectiveness of electron scavenging at $273 \mathrm{~K}$ is much larger than at $130 \mathrm{~K}$, or

- irradiation destroys type B traps.

If the faster component of exponential rise seen in fresh samples at $130 \mathrm{~K}$ is correlated with the population of traps type $\mathrm{B}$, the destruction of such traps during irradiation seems to be more probable. 


\section{Hexadecanol}

Measurements of $o$-Ps formation stability in time were also performed for one of alkane derivatives, i.e. $n$-hexadecanol- $1, \mathrm{C}_{16} \mathrm{H}_{33} \mathrm{OH}$. The procedure was like previously: the sample was molten (melting point $322 \mathrm{~K}$ ), degassed and cooled to $130 \mathrm{~K}$. Then the series of lifetime spectra was taken. No changes of $I_{3}$ in time were observed. One can try to explain the lack of effect by activation of molecular motions reducing the size of traps accommodating electrons, the same way as it occurs in alkanes above $200 \mathrm{~K}$. Thus, we tried to produce larger size free volumes by introduction of an admixture of shorter molecules, i.e. the measurements were performed for $n$-octadecanol- $1+10 \%$ of $n$-hexadecanol- 1 . However, also in that case the $o$-Ps intensity was stable, not rising with time. Another proposed source of $I_{3}$ stability in time can be the polar nature of alkanols. In a polar medium the potential trap for electron can be deeper and the energy needed to pick the electron larger than that available after binding electron and positron into Ps atom.

\section{Conclusions}

In alkanes, like in many polymers, the intensity of positronium formation changes in time. The data collected in this paper suggest that there are two kinds of traps, accommodating the excess electrons: the ones, which are emptied by thermal treatment at the temperatures above $200 \mathrm{~K}$, the other ones which keep electrons up to the temperature of transition to the rotator phase. Disappearance of $I_{3}$ surplus after several days of irradiation at $273 \mathrm{~K}$ we explain tentatively as due to the destruction of "type B" traps. The nature of these traps is for us unknown yet. The other explanation of that disappearance, i.e. as the result of very effective scavenging by radicals at elevated temperature, can be checked by performing the series of $I_{3}$ measurements as a function of time at fixed intermediate temperatures, from $140 \mathrm{~K}$ to $270 \mathrm{~K}$.

\section{Acknowledgments}

Authors wish to thank Prof. T. Goworek for the suggestion of this experiment and for helpful discussions. We are also grateful to Dr J. Wawryszczuk for technical assistance.

\section{References}

[1] O.E. Mogensen, J. Chem. Phys. 60, 998 (1974).

[2] V.M. Byakov, V.I. Goldanskii, V.P. Shantarovich, Dokl. Akad. Nauk SSSR 219, 633 (1974).

[3] P. Kindl, G. Reiter, Phys. Status Solidi A 104, 707 (1987).

[4] C. He, T. Suzuki, V.P. Shantarovich, L. Ma, M. Matsuo, K. Kondo, Y. Ito, Phys. Lett. A 313, 223 (2003).

[5] T. Hirade, Radiat. Phys. Chem. 68, 375 (2003). 
[6] Y. Ito, T. Hirade, E. Hamada, T. Suzuki, Y. Ito, Acta Phys. Pol. A 95, 533 (1999).

[7] T. Hirade, F.H.J. Maurer, M. Eldrup, Radiat. Phys. Chem. 58, 465 (2000).

[8] C. He, V.P. Shantarovich, T. Suzuki, S.V. Stepanov, R. Suzuki, M. Matsuo, J. Chem. Phys. 122, 214907 (2005).

[9] S.V. Stepanov, V.M. Byakov, C. He, T. Hirade, K.V. Mikhin, V.I. Grafutin, in: Proc. 7th Int. Moscow School of Physics (32nd ITEP Winter School of Physics), Eds. A.L. Suvorov, F.S. Dzheparov, M.A. Kozodaev, S.V. Stepanov, Academprint, Moscow 2004, p. 183.

[10] J. Kansy, Nucl. Instrum. Methods A 374, 235 (1996).

[11] T. Goworek, J. Wawryszczuk, R. Zaleski, B. Zgardzińska, to be published in Radiat. Phys. Chem. 